\title{
Plug-and-Play Control in Islanded AC Microgrids with Flexible Structures
}

\author{
Mahdieh S. Sadabadi \\ Dept. of Automatic Control and Systems Eng. \\ University of Sheffield \\ Sheffield, United Kingdom \\ m.sadabadi@sheffield.ac.uk
}

\author{
Zhengyu Lin \\ School of Mechanical, Electrical and Manufacturing Eng. \\ Loughborough University \\ Loughborough, United Kingdom \\ z.lin@lboro.ac.uk
}

\begin{abstract}
This paper proposes a plug-and-play decentralized control approach for voltage and frequency stabilization in islanded AC microgrids consisting of multiple power-electronicsinterfaced distributed generation (DG) units. The proposed control strategy guarantees stability and desired $\mathscr{H}_{\infty}$ level in voltage tracking performance of islanded microgrids. The line-independent feature of the proposed voltage control scheme facilitates the plug-and-play operation of DG units. The simulation results, carried out in MATLAB/Simscape Electrical environment, demonstrate the suitability of the proposed voltage control technique for islanded AC microgrids with flexible structures.
\end{abstract}

Keywords: AC microgrids, Voltage stabilization, Plug-andplay control, $\mathscr{H}_{\infty}$ control.

\section{INTRODUCTION}

Arbitrary plug-in and plug-out of distributed generation (DG) units in islanded inverter-interfaced microgrids pose a challenging issue due to uncertainty in microgrid structures and complexity in control design [1]. The existing control approaches for islanded microgrids can be categorized as droop-based and non-droop-based control strategies referred to as plug-and-play $(\mathrm{PnP})$ control.

Droop control offers several advantages in terms of simple structure, ease of implementation, scalability, sparse communication; however, it suffers from poor transient behaviour and instability issues to large and fast load changes as well as dependency on line dynamics [2]. In non-droop-based control techniques, e.g., [1], [3], [4], frequency of islanded microgrids is controlled based on the accurate synchronization of internal oscillators in an open-loop manner [5], whereas voltage magnitude is regulated using a closed-loop control system. In [4], the PnP operation of DG units has been modeled as polytopic uncertainties. However, a large number of vertices in the polytope leads to high computational burdens and complexity. The main drawback of the proposed PnP voltage control approaches presented in [1], [3] is that they do not guarantee desired transient behaviour in the voltage tracking performance of islanded $\mathrm{AC}$ microgrids and might result in poor system-wide performance during the $\mathrm{PnP}$ operations of DG units. Hence, it is essential to develop a systematic PnP voltage control approach, which guarantees both stability and smooth transient performance of islanded microgrids- this facilitates the seamless integration and/or removal of DG units from islanded microgrids without control redesigning.

Motivated by the aforementioned challenges and the limitation of the existing approaches, this paper develops a $\mathrm{PnP}$ voltage control approach that ensures the stable operation and satisfactory performance of microgrids. To this end, the design of decentralized PnP local voltage controllers is formulated as a loop shaping $\mathscr{H}_{\infty}$ control problem, mathematically described as a convex optimization problem with Linear Matrix Inequality (LMI) constraints. The solution of the optimization problem is expressed in terms of a decentralized two-degreeof-freedom (2DOF) feedback-feedforward local voltage control that guarantees the stability of islanded microgrids and provides satisfactory performance in terms of voltage-tracking transient behaviour. Contrary to the $\mathrm{PnP}$ voltage stabilization methods in [1], [2], the proposed voltage control technique in this paper is line-independent and does not require the knowledge of power lines connecting DG units. Moreover, unlike the existing PnP control approaches in [1], [3], the proposed controller guarantees desired voltage transient performance specifications. The paper describes theoretical aspects involved in the design procedure of the local voltage controllers in islanded AC microgrids and evaluates the performance of the designed controllers based on simulation case studies in MATLAB/Simscape Electrical. The simulation results confirm the satisfactory performance of the proposed voltage control strategy in the voltage regulation, as well as robustness to $\mathrm{PnP}$ functionality of DG units.

The paper is organized as follows. The dynamic model of islanded AC microgrids with power-electronics-interfaced DG units is given in Section II. The proposed line-independent PnP $\mathscr{H}_{\infty}$ voltage control strategy is presented in Section IV. The simulation results are provided in Section IV. Finally, the paper ends with concluding remarks in Section V.

Notation: The notation used in this paper is standard. In particular, $\mathbf{0}_{n \times m}$ is an $n \times m$ matrix of zeros and $\mathbf{I}_{n}$ is an $n \times n$ Identity matrix. The symbol $\operatorname{diag}\left(x_{1}, \ldots, x_{n}\right)$ indicates a blockdiagonal matrix whose diagonal block matrices are $x_{i}$. For a symmetric matrix $X$, the positive definite and negative definite operators are respectively shown by $X \succ 0$ and $X \prec 0$. 


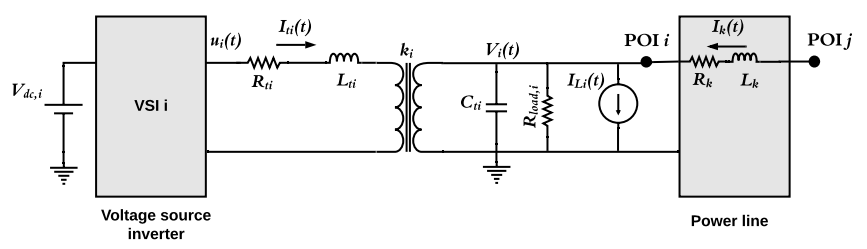

Fig. 1. A schematic single line diagram of an inverter-interfaced DG unit.

\section{Dynamic Model of Islanded AC Microgrids}

In this section, a dynamic model for an islanded AC microgrid consisting of $n$ arbitrarily connected DG units through $m$ power lines is presented. Each DG is composed of a voltage source inverter (VSI), an RL-filter, a shunt capacitor, a stepup transformer with a transformation ratio $\kappa_{i}$, and local loads connected at the point of interconnections (POIs). The source side of DG units is simplified as an ideal DC voltage source. The single line diagram of each DG unit is shown in Fig. 1. In this figure, $\left(R_{t_{i}}, L_{t_{i}}\right)$ are the filter parameters of the $i^{t h}$ inverter, $V_{d c, i}$ is the VSC DC side voltage, $\kappa_{i}$ is the transformer ratio, $C_{t_{i}}$ is a capacitance, and $\left(R_{k}, L_{k}\right)$ are the parameters of the distribution line $k$.

Under balanced conditions, the dynamics of an islanded microgrid is described by the following dynamical equations in direct and quadrature $(d q)$ frame [1]:

$$
\begin{aligned}
C_{t_{i}} \dot{V}_{i}(t) & =\left(-\frac{1}{R_{\text {load }, i}} \mathbf{I}_{2}+\omega_{0} C_{t_{i}} \mathbf{J}\right) V_{i}(t)+\kappa_{i} I_{t_{i}}(t)-I_{L_{i}}(t) \\
& -\sum_{k=1}^{m} \mathscr{B}_{i k} I_{k}(t), \\
L_{t_{i}} \dot{I}_{t_{i}}(t) & =-\kappa_{i} V_{i}(t)+\left(-R_{t_{i}} \mathbf{I}_{2}+\omega_{0} L_{t_{i}} \mathbf{J}\right) I_{t_{i}}(t)+u_{i}(t), \\
L_{k} \dot{I}_{k}(t)= & \left(-R_{k} \mathbf{I}_{2}+\omega_{0} L_{k} \mathbf{J}\right) I_{k}(t)+\sum_{i=1}^{n} \mathscr{B}_{i k} V_{i}(t) .
\end{aligned}
$$

for $i=1, \ldots, n$, where $\mathbf{J}=\left[\begin{array}{cc}0 & 1 \\ -1 & 0\end{array}\right], \mathbf{I}_{2}=\left[\begin{array}{ll}1 & 0 \\ 0 & 1\end{array}\right], V_{i}(t) \in$ $\mathbb{R}^{2 \times 1}, I_{t_{i}}(t) \in \mathbb{R}^{2 \times 1}, I_{L_{i}} \in \mathbb{R}^{2 \times 1}, u_{i}(t) \in \mathbb{R}^{2 \times 1}$, and $I_{k}(t) \in \mathbb{R}^{2 \times 1}$ are the $d q$ components of voltage at POI $i$, DG current, load current, VSC terminal voltage, and distribution line current, respectively. The loads at the POIs are modeled as a constant resistance $\left(R_{\text {load }, i}\right)$ and a local load with current $I_{L_{i}}$ that is topologically and parametrically unknown. The term $\mathscr{B}_{i k}$ defines the direction of the power line current, i.e., $\mathscr{B}_{i k}=1$ if line $k$ leaves DG $i, \mathscr{B}_{i k}=-1$ if line $k$ enters DG $i$, and $\mathscr{B}_{i k}=0$ otherwise.

\section{Voltage Control of Multi-DG Microgrids}

It is assumed that the frequency of each DG is dictated by an internal oscillator with frequency $\omega_{0}=2 \pi f_{0}$, where $f_{0}$ is the nominal frequency of the system. The oscillators are synchronized through communication systems [5]. The islanded microgrid is equipped with a hierarchical voltage control block composed of a higher-level control system with a centralized structure and local voltage controllers of each DG.
The main function of the higher-level control is to maintain optimal and efficient operation of the microgrids [5]. The higher-level control also determines and transmits appropriate voltage setpoints $V_{i}^{*}=\left[\begin{array}{ll}V_{i, d}^{*} & V_{i, q}^{*}\end{array}\right]^{T}$ to the local voltage controller of DG units. The local voltage controllers measure the voltage at their corresponding POIs and then provide the voltage tracking according to the reference setpoints [2]. The main focus of this paper is on the design of local voltage controllers so that each DG robustly stabilizes voltages at its own POI, and provides offset-free tracking of its voltage setpoints determined by the higher-level controller.

\section{A. Decentralized Voltage Control}

The main goal of the local voltage controllers of DG units is to guarantee the voltage stability and offset-free tracking of reference voltages $V_{i}^{*}$ [1]. In order to provide an offset-free tracking, it is assumed that voltage controllers are in terms of a multivariable two-degree-of-freedom (2DOF) control and have a fully decentralized configuration that does not require any communication and information exchange. The local control law for DG $i, i=1, \ldots, n$ is structured as follows:

$$
\begin{aligned}
& u_{i}(t)=K_{i_{1}} V_{i}(t)+K_{i_{2}} I_{i_{i}}(t)+K_{i_{3}} v_{i}(t)+K_{f_{i}} V_{i}^{*}, \\
& \dot{v}_{i}(t)=-V_{i}(t)+V_{i}^{*},
\end{aligned}
$$

where $K_{i}=\left[\begin{array}{lll}K_{i_{1}} & K_{i_{2}} & K_{i_{3}}\end{array}\right] \in \mathbb{R}^{2 \times 6}$ is the feedback control gain and $K_{f_{i}} \in \mathbb{R}^{2 \times 2}$ is the feedforward control matrix that are designed such that the stability and the desired performance of islanded inverter-interfaced microgrids is guaranteed.

\section{B. Voltage Tracking Performance Specification}

To achieve the desired voltage tracking performance specification according to IEEE standards in [6], the $\mathscr{L}_{2}$-gain of the error signal $e_{i}(t)=V_{i}^{*}-V_{i}(t)$ to the reference voltage $V_{i}^{*}$ should be minimized. To this end, the desired voltage tracking performance of islanded microgrids is formulated as a loop shaping problem, by shaping the closed-loop sensitivity transfer function. To achieve a certain closed-loop bandwidth and limit the impact of load disturbances on the voltage tracking error, the following $\mathscr{H}_{\infty}$ optimization problem is considered:

$$
\begin{aligned}
& \min \gamma \\
& \text { subject to } \quad\|W(s) S(s)\|_{\infty}<\gamma
\end{aligned}
$$

where $S(s)=\left(\mathbf{I}_{2 n}+G(s) \mathbf{K}\right)^{-1}$ is the sensitivity function [7] (page 22), $G(s)$ is the open-loop transfer function between $V_{t}(t)$ and $V(t), \mathbf{K}=\operatorname{diag}\left(K_{1}, \ldots, K_{n}\right)$, and $W(s)=$ $\operatorname{diag}\left(W_{1}(s), \ldots, W_{n}(s)\right)$ is a weighting filter. The low-pass filter $W_{i}(s)$ is designed based on desired reference tracking performance as follows:

$$
W_{i}(s)=\frac{\frac{s}{M_{i}}+\omega_{i}^{*}}{s+\omega_{i}^{*} \varepsilon_{i}} \mathbf{I}_{2}
$$

where $\omega_{i}^{*}$ is the desired closed-loop bandwidth, $\varepsilon_{i}$ is the maximum steady-state error, and $M_{i} \leq 2$ to ensure sufficient 
module margin [7]. The state-space realization of the low-pass filter $W_{i}(s)$ is represented as follows:

$$
W_{i}(s):\left\{\begin{array}{l}
\dot{v}_{i}(t)=A_{w_{i}} v_{i}(t)+B_{w_{i}} e_{V_{i}}(t), \\
z_{i}(t)=C_{w_{i}} v_{i}(t)+D_{w_{i}} e_{V_{i}}(t),
\end{array}\right.
$$

where $e_{V_{i}}(t)=-V_{i}(t)+V_{i}^{*}$ is the voltage error signal, $z_{i}(t)$ is a performance output that is used to satisfy the loop-shaping characteristics of microgrid dynamics, and $A_{w_{i}} \in \mathbb{R}^{2 \times 2}, B_{w_{i}} \in$ $\mathbb{R}^{2 \times 2}, C_{w_{i}} \in \mathbb{R}^{2 \times 2}$, and $D_{w_{i}} \in \mathbb{R}^{2 \times 2}$ are obtained from the transfer function $W_{i}(s)$ given in (4). The state-space realization of $W(s) S(s)$ with the decentralized 2DOF voltage control strategy in (2) is obtained as follows:

$$
\begin{aligned}
\dot{x}_{i}(t) & =\left(A_{i}+B_{i} K_{i}\right) x_{i}(t)+\left(B_{v_{i}}+B_{i} K_{f_{i}}\right) V_{i}^{*}+B_{l_{i}} \sum_{k=1}^{m} \mathscr{B}_{i k} I_{k}(t), \\
z_{i}(t) & =C_{i} x_{i}(t)+D_{v_{i}} V_{i}^{*} .
\end{aligned}
$$

where $x_{i}(t)=\left[\begin{array}{lll}V_{i}^{T}(t) & I_{t_{i}}^{T}(t) & v_{i}^{T}(t)\end{array}\right]^{T}$ and

$$
\begin{aligned}
A_{i} & =\left[\begin{array}{c|c}
A_{g_{i}} & \mathbf{0}_{2 \times 2} \\
\hline B_{w_{i}} C_{g_{i}} & A_{w_{i}}
\end{array}\right], B_{i}=\left[\begin{array}{c}
\mathbf{0}_{2 \times 2} \\
\frac{1}{L_{t_{i}}} \mathbf{I}_{2} \\
\hline \mathbf{0}_{2 \times 2}
\end{array}\right], B_{v_{i}}=\left[\begin{array}{c}
\mathbf{0}_{4 \times 2} \\
\hline-B_{w_{i}}
\end{array}\right], \\
B_{l_{i}} & =\left[\begin{array}{c}
-\frac{1}{C_{t_{i}}} \mathbf{I}_{2} \\
\mathbf{0}_{4 \times 2}
\end{array}\right], D_{v_{i}}=-D_{w_{i}}, C_{i}=\left[\begin{array}{ll}
D_{w_{i}} C_{g_{i}} & C_{w_{i}}
\end{array}\right],
\end{aligned}
$$

where

$$
\begin{aligned}
A_{g_{i}} & =\left[\begin{array}{cccc}
-\frac{1}{R_{\text {load }, i} C_{t_{i}}} & \omega_{0} & \frac{\kappa_{i}}{C_{t_{i}}} & 0 \\
-\omega_{0} & -\frac{1}{R_{\text {load }, i} C_{t_{i}}} & 0 & \frac{\kappa_{i}}{C_{t_{i}}} \\
-\frac{\kappa_{i}}{L_{t_{i}}} & 0 & -\frac{R_{t_{i}}}{L_{t_{i}}} & \omega_{0} \\
0 & -\frac{\kappa_{i}}{L_{t_{i}}} & -\omega_{0} & -\frac{R_{t_{i}}}{L_{t_{i}}}
\end{array}\right], \\
C_{g_{i}} & =\left[\begin{array}{ll}
\mathbf{I}_{2} & \mathbf{0}_{2 \times 2}
\end{array}\right] .
\end{aligned}
$$

C. Proposed Line-Independent PnP $\mathscr{H}_{\infty}$ Voltage Control Design

This subsection addresses the scalable design of local voltage controllers for achieving $\mathscr{H}_{\infty}$ voltage-tracking performance specification in islanded inverter-interfaced microgrids. The proposed control approach is formulated by a set of linear matrix inequalities (LMIs) as presented in the following theorem.

Theorem 1. If voltage control gains $\left(K_{i}, K_{f_{i}}\right)$ in (2), $i=$ $1, \ldots, n$, are designed using the following conditions:

$$
\left[\begin{array}{ccc}
A_{i} Y_{i}+Y_{i} A_{i}^{T}+B_{i} H_{i}+H_{i}^{T} B_{i}^{T} & \left(B_{v_{i}}+B_{i} K_{f_{i}}\right) & \left(C_{i} Y_{i}\right)^{T} \\
\left(B_{v_{i}}+B_{i} K_{f_{i}}\right)^{T} & -\gamma_{i}^{2} \mathbf{I}_{2} & D_{v_{i}}^{T} \\
C_{i} Y_{i} & D_{v_{i}} & -\mathbf{I}_{2}
\end{array}\right] \prec 0
$$

where $H_{i} \in \mathbb{R}^{2 \times 6}$ and $\gamma_{i}>0$, with the following fixed-structure $Y_{i} \succ 0$

$$
Y_{i}=\left[\begin{array}{c|c}
\frac{1}{\eta C_{t_{i}}} \mathbf{I}_{2} & \mathbf{0}_{2 \times 4} \\
\hline \mathbf{0}_{4 \times 2} & \mathscr{Y}_{i}
\end{array}\right], \quad \mathscr{Y}_{i} \succ 0
$$

where $\eta>0$ and $\mathscr{Y}_{i} \in \mathbb{R}^{4 \times 4}$, the overall microgrid in (6) is asymptotically stable and $\|W(s) S(s)\|_{\infty} \leq \gamma$, where $\gamma=$ $\max _{i=1, \ldots, n} \gamma_{i}$.

Proof. See Appendix in Section VI.

The feedback and feedforward voltage control gains in (2) are obtained via a solution of the following convex optimization problem subject to a set of linear matrix inequalities (LMIs):

$$
\min _{\eta, H_{i}, \mathscr{Y}_{i}, K_{f_{i}}} \gamma_{i}
$$

subject to

$$
\begin{gathered}
{\left[\begin{array}{ccc}
A_{i} Y_{i}+Y_{i} A_{i}^{T}+B_{i} H_{i}+H_{i}^{T} B_{i}^{T} & \left(B_{v_{i}}+B_{i} K_{f_{i}}\right) & \left(C_{i} Y_{i}\right)^{T} \\
\left(B_{v_{i}}+B_{i} K_{f_{i}}\right)^{T} & -\gamma_{i}^{2} \mathbf{I}_{2} & D_{v_{i}}^{T} \\
C_{i} Y_{i} & D_{v_{i}} & -\mathbf{I}_{2}
\end{array}\right] \prec 0,} \\
Y_{i}=\left[\begin{array}{c|c}
\frac{1}{\eta C_{t_{i}}} \mathbf{I}_{2} & \mathbf{0}_{2 \times 4} \\
\hline \mathbf{0}_{4 \times 2} & \mathscr{Y}_{i}
\end{array}\right] \succ 0 .
\end{gathered}
$$

for $i=1, \ldots, n$. By solving the above optimization problem, the feedforward gain matrix $K_{f_{i}}$ is obtained. Moreover, the feedback gain of the voltage control is expressed as $K_{i}=H_{i} Y_{i}^{-1}$.

Remark 1. According to the optimization problem in (11), the design of the local dq-based voltage controller for each $D G$ unit is independent of the parameters of other DG units and power lines. This facilities the plug-and-play operation of DG units. More specifically, when DG $j$, with an $\mathscr{H}_{\infty}$ performance level $\gamma_{j}$ is unplugged, the stability of microgrids and their performance with the $\mathscr{H}_{\infty}$ performance level of $\max _{i=1, \ldots, n, i \neq j} \gamma_{i}$ are preserved. Moreover, when a new $D G$, let say $D G n+1$ is plugged into an islanded AC microgrid, the microgrid remains stable. Furthermore, the $\mathscr{H}_{\infty}$ performance level changes to $\max \left(\gamma, \gamma_{n+1}\right)$, provided that the new DG unit with the proposed $2 D O F$ voltage controller achieves an $\mathscr{H}_{\infty}$ performance level of $\gamma_{n+1}$.

\section{Proposed 2DOF Voltage Control Design Algorithm}

In this subsection, the results presented in this paper are summerized and a voltage control design algorithm for powerelectronics-interfaced DG units in an islanded AC microgrid is introduced.

According to Remark 1, the main feature of the proposed voltage control strategy in this paper is that the design of DG units' voltage controllers are independent of power lines in microgrids. The algorithm is summarized as follows:

Proposed Algorithm for the Design of Line-Independent PnP $\mathscr{H}_{\infty}$ Voltage Controllers in Islanded Microgrids:

1) Weighting filter design: The weighting filter $W_{i}(s)$ is structured by (4).

2) Voltage control structure: The voltage controller for each DG unit is structured according to the control law in (2).

3) Design of control gains $\left(K_{i}, K_{f_{i}}\right)$ in (2): The control gains $\left(K_{i}, K_{f_{i}}\right)$ are designed by a solution of the convex optimization problem in (11). 


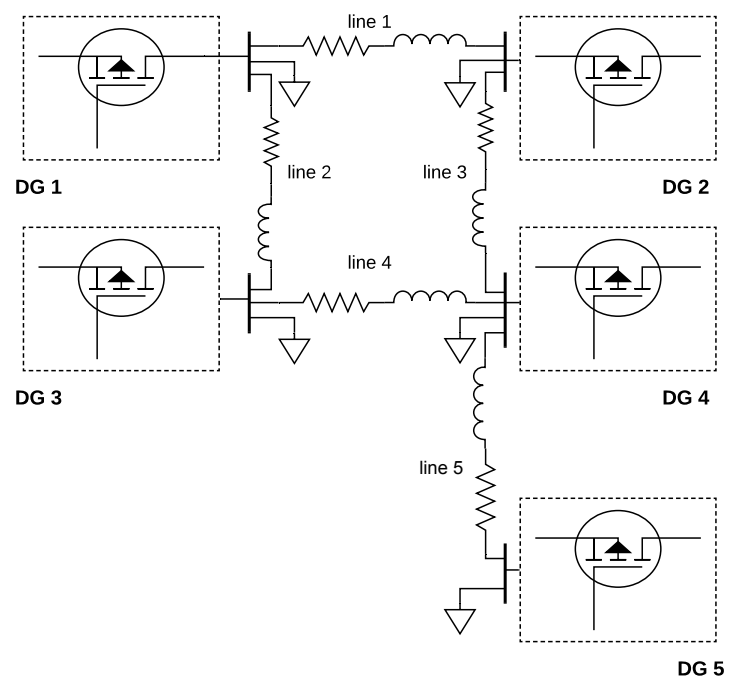

Fig. 2. Layout of an islanded inverter-interfaced AC microgrid consisting of $n=5$ DG units connecting by $m=5$ power lines.

TABLE I

PARAMETERS OF THE ISLANDED MICROGRID UNDER STUDY IN FIG. 2.

\begin{tabular}{cccccc}
\hline \multicolumn{7}{c}{ Filter parameters \& dq voltage setpoints } \\
DG 1 & $R_{t}(m \Omega)$ & $L_{t}(\mu H)$ & $C_{t}(\mu F)$ & $V_{d}^{*}(\mathrm{pu})$ & $V_{q}^{*}(\mathrm{pu})$ \\
DG 2 & 1.2 & 93.2 & 62.86 & 0.866 & 0.5 \\
DG 3 & 1.6 & 94.8 & 60 & 0.866 & -0.5 \\
DG 4 & 1.5 & 107.7 & 65.86 & 0.8 & -0.6 \\
DG 5 & 1.5 & 90.6 & 62.86 & 0.8 & 0.6 \\
\hline \multicolumn{7}{c}{0.8} \\
\hline \multicolumn{7}{c}{ VSC, transformer parameters, and base voltage } \\
$V_{d c}=$ 2000 V & $f_{0}=60 \mathrm{~Hz}$ & $\kappa=0.6 / 13.8$ & $V_{\text {base }}=500 \mathrm{~V}$ \\
Line impedances \& Load parameters \\
Line 2 and Load 1 \\
Line 3 and Load 3 & $R_{k}(\Omega)$ & $L_{k}(\mu H)$ & $R_{\text {load }, i}(\Omega)$ & $L_{\text {load }, i}(m H)$ & $r_{i}(\Omega)$ \\
Line 4 and Load 4 & 1 & 0.9 & 93 & 123.1 & 0.1 \\
Line 5 and Load 5 & 1.2 & 0.4 & 85 & 134.3 & 0.1 \\
\hline
\end{tabular}

The convex optimization problems in (11) can easily be solved using YALMIP [8] as an interface and MOSEK [9] as a solver.

\section{Simulation Results}

The performance of the proposed plug-and-play 2DOF voltage control approach in Section IV is illustrated by a case study of an islanded microgrid with mesh topology composed of $n=5$ power-electronics-interfaced DG units connected through $m=5$ distribution lines, as depicted in Fig. 2. The topology and parameters of the microgrid under study are respectively shown in Fig. 2 and Table I. The load at POI $i$; $i=1, \ldots, 5$, includes a parallel $R L$ load with a load resistance $\left(R_{\text {load }, i}\right)$, a load inductance $\left(L_{\text {load }, i}\right)$, and the parasitic resistance of the load inductance $\left(r_{i}\right)$. The simulation case studies are conducted in MATLAB/Simscape Electrical environment.

\section{A. Performance Evaluation}

It is assumed that DG 5 is initially disconnected from the islanded microgrid in Fig. 2. To verify the performance of the proposed voltage control technique in the voltage regulation and the robustness properties in the presence of load variation, unknown load dynamics, and the plug-and-play operation of DG units, the following case studies are carried out:

1) Voltage Tracking: The $d$ and $q$ components of the voltage setpoint for DG 1 at POI 1 change from $(0.866,0.5) \mathrm{pu}$ to $(0.5,0.866) \mathrm{pu}$ at $t=0.5 \mathrm{~s}$.

2) Robustness to Load Uncertainties: The load conductance of DG 2 suddenly decreases by $40 \%$ at $t=0.75 s$.

3) PnP Functionality of DG Units: DG 5 is plugged into the microgrid in Fig. 2 at $t=1.0 \mathrm{~s}$ and connected to DG 4 via the power line 5 . Then, it is plugged-out from the microgrid at $t=1.25 \mathrm{~s}$.

4) Robustness to Unknown Load Dynamics: A three-phase passive load with unknown dynamics is connected in parallel with the load at POI 2 at $t=1.5 \mathrm{~s}$.

Fig. 3 shows the dynamic responses of all DG units due to the above mentioned scenarios. From this figure, one can observe that the local voltage controllers track the reference setpoints at POIs with a rise time of about two/three cycles of $f_{0}$ and with no overshoot. Furthermore, the effect of voltage changes on the voltage signals of POI 2, POI 3, and POI 4 is negligible. Moreover, the results illustrate the performance of local voltage controllers to the load uncertainty and the plug-and-play operation of DG units.

\section{B. A Comparative Case Study}

The performance of the proposed voltage control strategy, in terms of voltage stability and robustness to line failure, is compared with the one in [1]. To this end, it is assumed that $d$ component of the voltage setpoint at POI 1 is stepped down to $0.5 \mathrm{pu}$ at $t=1.5 \mathrm{~s}$, while the $q$ component is stepped up to $0.866 \mathrm{pu}$ at the same time. Furthermore, due to a line failure, the line 1 connecting DG 1 to DG 2 is disconnected at $t=3 \mathrm{~s}$. The dynamic responses of DG 1 and DG 2 are depicted in Fig. 4. In order to observe the transient performance of both approaches, only $d$ and $q$ components of the voltages are shown.

The results of Fig. 4 reveal that the proposed voltage control strategy provides superior dynamic performance in terms of voltage tracking and robustness to uncertainties affecting the topology of islanded AC microgrids.

\section{CONCLUSION}

This paper develops a plug-and-play voltage control approach that guarantees stability and $\mathscr{H}_{\infty}$ performance specifications in islanded microgrids. In the proposed control approach, the design of the local voltage controllers for DG units is carried out in a scalable manner, i.e., the local voltage control design does not rely on the parameters of power lines connecting different DG units. The main advantages of the proposed voltage control scheme are that: (i) it is fully decentralized and independent of the parameters of power lines and (ii) the proposed voltage control approach ensures stability and offset-free voltage tracking in islanded microgrids with flexible structures. The performance of the proposed control strategy 
(a)

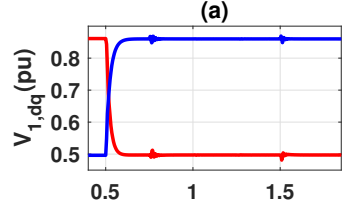

(e)
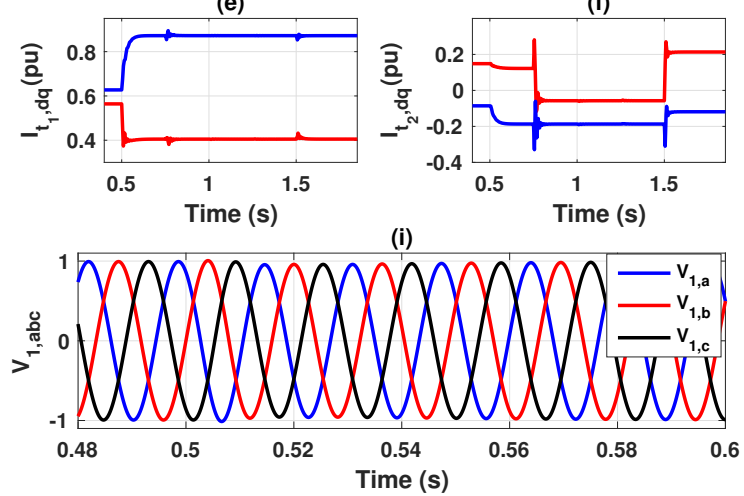

(b)

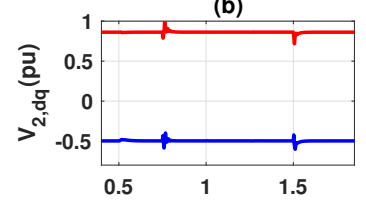

(f)

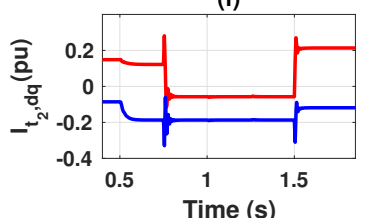

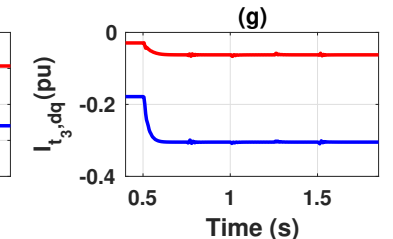

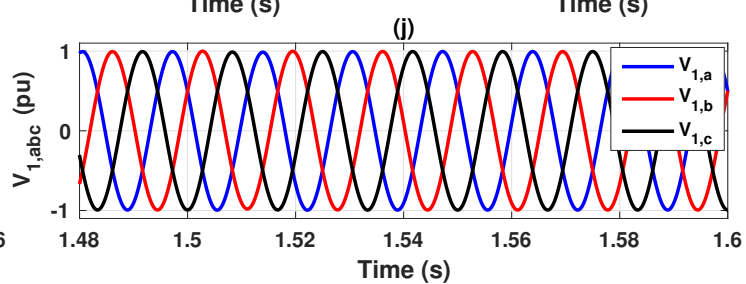

Fig. 3. Dynamic response of DG1-DG4: (a)-(d) $d$ component (red) and $q$ component (blue) of voltage signals at POIs, (e)-(h) $d$ component (red) and $q$ component (blue) of filter current trajectories $\left(I_{t_{i}}\right)$, and (i)-(j) instantaneous voltage at POI 1 for different time periods (zoomed version).
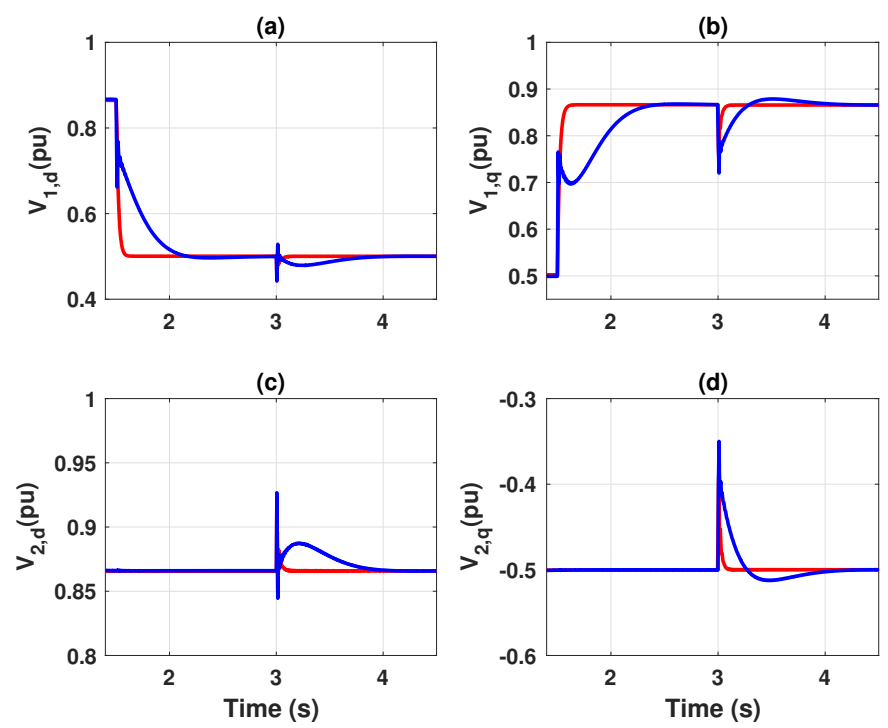

Fig. 4. Performance of the proposed PnP voltage control (red) and the controller of [1] (blue) due to setpoint changes at $t=1.5 \mathrm{~s}$ and a line failure at $t=3 \mathrm{~s}$ : (a) $d$ component of the voltage signal at POI 1 , (b) $q$ component of the voltage signal at POI 1 , (c) $d$ component of the voltage signal at POI 2 , and (d) $q$ component of the voltage signal at POI 2.

is verified under several case studies in MATLAB/Simscape software.

\section{APPENDIX: PROOF OF THEOREM 1}

Proof. We propose the following common quadratic Lyapunov function for overall AC microgrids modeled by (6):

$$
\mathscr{V}=\sum_{i=1}^{n} x_{i}^{T}(t) P_{i} x_{i}(t)+\eta \sum_{k=1}^{m} I_{k}^{T}(t) L_{k} I_{k}(t),
$$

where $P_{i} \succ 0$ is structured as follows:

$$
P_{i}=Y_{i}^{-1}=\left[\begin{array}{c|c}
\eta C_{t_{i}} \mathbf{I}_{2} & \mathbf{0}_{2 \times 4} \\
\hline \mathbf{0}_{4 \times 2} & \mathscr{Y}_{i}^{-1}
\end{array}\right],
$$

Note that $\mathscr{V} \geq 0$ and $\mathscr{V}(0)=0$. The time derivative of $\mathscr{V}$ along the closed-loop trajectories in (6) is obtained as follows:

$$
\begin{aligned}
\dot{\mathscr{V}} & =\sum_{i=1}^{n} x_{i}^{T}(t) Q_{i} x_{i}(t) \\
& +\sum_{i=1}^{n}\left(x_{i}^{T}(t) P_{i} B_{I_{i}} \sum_{k=1}^{m} \mathscr{B}_{i k} I_{k}(t)+\sum_{k=1}^{m} I_{k}^{T}(t) \mathscr{B}_{i k} B_{I_{i}}^{T} P_{i} x_{i}(t)\right) \\
& +\sum_{i=1}^{n} x_{i}^{T}(t) P_{i}\left(B_{v_{i}}+B_{i} K_{f_{i}}\right) V_{i}^{*}+\sum_{i=1}^{n} V_{i}^{* T}\left(B_{v_{i}}+B_{i} K_{f_{i}}\right)^{T} P_{i} x_{i}(t) \\
& -2 \eta \sum_{k=1}^{m} I_{k}^{T}(t) R_{k} I_{k}(t)+\eta\left(V^{T}(t) \mathscr{B} I(t)+I^{T}(t) \mathscr{B}^{T} V(t)\right),
\end{aligned}
$$

where $Q_{i}=\left(A_{i}+B_{i} K_{i}\right)^{T} P_{i}+P_{i}\left(A_{i}+B_{i} K_{i}\right)$. By direct calculation and invoking the specific structure of $P_{i}$ in (13), it yields that

$$
\begin{gathered}
\sum_{i=1}^{n}\left(x_{i}^{T}(t) P_{i} B_{I_{i}} \sum_{k=1}^{m} \mathscr{B}_{i k} I_{k}(t)+\sum_{k=1}^{m} I_{k}^{T}(t) \mathscr{B}_{i k} B_{I_{i}}^{T} P_{i} x_{i}(t)\right) \\
+\eta\left(V^{T}(t) \mathscr{B} I+I^{T}(t) \mathscr{B}^{T} V\right)=0 .
\end{gathered}
$$


Therefore, $\dot{\mathscr{V}}$ in (14) can be simplified as follows:

$$
\begin{aligned}
\dot{\mathscr{V}} & =\sum_{i=1}^{n} x_{i}^{T}(t) Q_{i} x_{i}(t)-2 \eta \sum_{k=1}^{m} I_{k}^{T}(t) R_{k} I_{k}(t) \\
& +\sum_{i=1}^{n} x_{i}^{T}(t) P_{i}\left(B_{v_{i}}+B_{i} K_{f_{i}}\right) V_{i}^{*} \\
& +\sum_{i=1}^{n} V_{i}^{* T}\left(B_{v_{i}}+B_{i} K_{f_{i}}\right)^{T} P_{i} x_{i}(t) .
\end{aligned}
$$

Since $\eta>0$ and $\sum_{k=1}^{m} I_{k}^{T}(t) R_{k} I_{k}(t)>0, \forall t \geq 0$, one obtains that

$$
\dot{\mathscr{V}} \leq \sum_{i=1}^{n}\left[\begin{array}{c}
x_{i}(t) \\
V_{i}^{*}
\end{array}\right]^{T}\left[\begin{array}{cc}
Q_{i} & P_{i}\left(B_{v_{i}}+B_{i} K_{f_{i}}\right) \\
\left(B_{v_{i}}+B_{i} K_{f_{i}}\right)^{T} P_{i} & 0
\end{array}\right]\left[\begin{array}{c}
x_{i}(t) \\
V_{i}^{*}
\end{array}\right]
$$

It can be shown that the inequality condition in (9) is equivalent to the existence of a scalar $\varepsilon_{i}>0$ so that the following inequality holds:

$$
\begin{array}{r}
{\left[\begin{array}{c}
x_{i}(t) \\
V_{i}^{*}
\end{array}\right]^{T}\left[\begin{array}{cc}
Q_{i} & P_{i}\left(B_{v_{i}}+B_{i} K_{f_{i}}\right) \\
\left(B_{v_{i}}+B_{i} K_{f_{i}}\right)^{T} P_{i} & 0
\end{array}\right]\left[\begin{array}{c}
x_{i}(t) \\
V_{i}^{*}
\end{array}\right]} \\
\leq\left(-\varepsilon_{i} x_{i}^{T}(t) x_{i}(t)+\gamma_{i}^{2} V_{i}^{* T} V_{i}^{*}-z_{i}^{T}(t) z_{i}(t)\right)
\end{array}
$$

As a result,

$$
\dot{\mathscr{V}} \leq \sum_{i=1}^{n}\left(-\varepsilon_{i} x_{i}^{T}(t) x_{i}(t)+\gamma_{i}^{2} V_{i}^{* T} V_{i}^{*}-z_{i}^{T}(t) z_{i}(t)\right) .
$$

We define $\gamma=\max _{i=1 \ldots, n} \gamma_{i}$ and $\varepsilon=\min _{i=1, \ldots, n} \varepsilon_{i}$. Therefore, one obtains that

$$
\dot{\mathscr{V}} \leq-\varepsilon \mathbf{x}^{T}(t) \mathbf{x}(t)+\gamma^{2} \mathbf{V}^{* T} \mathbf{V}^{*}-\mathbf{z}^{T}(t) \mathbf{z}(t),
$$

where $\mathbf{x}(t)=\left[x_{1}^{T}(t), \ldots, x_{n}^{T}(t)\right]^{T}, \mathbf{V}^{*}=\left[V_{1}^{* T}, \ldots, V_{n}^{* T}\right]^{T}$, and $\mathbf{z}(t)=\left[z_{1}^{T}(t), \ldots, z_{n}^{T}(t)\right]^{T}$. According to [10], the inequality in (20) implies that $\|W(s) S(s)\|_{\infty} \leq \gamma$. This completes the proof of Theorem 1.

\section{REFERENCES}

[1] M. S. Sadabadi, Q. Shafiee, and A. Karimi, "Plug-and-play voltage stabilization in inverter-interfaced microgrids via a robust control strategy strategy," IEEE Transactions on Control Systems Technology, vol. 25, no. 3, pp. 781-791, May 2017.

[2] M. S. Sadabadi, A. Karimi, and H. Karimi, "Fixed-order decentralized/distributed control of islanded inverter-interfaced microgrids," Control Engineering Practice, vol. 45, pp. 174-193, Dec. 2015.

[3] M. Tucci and G. Ferrari-Trecate, "A scalable, line-independent control design algorithm for voltage and frequency stabilization in $\mathrm{AC}$ islanded microgrids," Automatica, vol. 111, pp. 1-7, Jan. 2020.

[4] M. ShafieeRad, Q. Shafiee, M. S. Sadabadi, and M. R. Jahed-Motlagh, "Decentralized voltage stabilization and robust performance satisfaction of islanded inverter-interfaced microgrids," IEEE Systems Journal, vol. 15, no. 2, pp. 1893-1904, June 2021.

[5] A. H. Etemadi, E. J. Davison, and R. Iravani, "A generalized decentralized robust control of islanded microgrids," IEEE Trans. Power Syst., vol. 29, no. 6, pp. 3102-3113, Nov. 2014.

[6] IEEE Std 2030.7, "IEEE standard for the specification of microgrid controllers," IEEE Power and Energy Society, Dec. 2017.

[7] S. Skogestad and I. Postlethwaite, Multivariable Feedback Control Analysis and design. Wiley, Second Edition, 2001.
[8] J. Löfberg, "YALMIP: A toolbox for modeling and optimization in MATLAB," in Proc. IEEE Int. Symp. Comp. Cont. Syst. Design (CACSD), 2004. [Online]. Available: http://control.ee.ethz.ch/ joloef/ yalmip.php

[9] MOSEK ApS, The MOSEK optimization software, 2011. [Online]. Available: http://www.mosek.com

[10] A. Isidori, Lectures in Feedback Design for Multivariable Systems. Springer, 2016. 